\title{
Common Fixed Points for Pairs of Mappings with Variable Contractive Parameters
}

\author{
J. R. Morales, ${ }^{1}$ E. M. Rojas, ${ }^{2}$ and Ravindra K. Bisht ${ }^{3}$ \\ ${ }^{1}$ Departamento de Matemáticas, Universidad de Los Andes, Mérida 5101, Venezuela \\ ${ }^{2}$ Departamento de Matemáticas, Pontificia Universidad Javeriana, Bogotá, Colombia \\ ${ }^{3}$ Department of Mathematics, Applied Sciences and Humanities, B. T. Kumaon Institute of Technology, \\ Dwarahat, Almora, Uttarakhand 262553, India
}

Correspondence should be addressed to E. M. Rojas; edixonr@gmail.com

Received 12 November 2013; Revised 15 January 2014; Accepted 15 January 2014; Published 2 March 2014

Academic Editor: Wei-Shih Du

Copyright (C) 2014 J. R. Morales et al. This is an open access article distributed under the Creative Commons Attribution License, which permits unrestricted use, distribution, and reproduction in any medium, provided the original work is properly cited.

We establish some common fixed point results for a new class of pair of contraction mappings having functions as contractive parameters, and satisfying minimal noncommutative operators property.

\section{Introduction and Preliminaries}

The metric fixed point theory has a vast literature; the Banach-Caccioppoli contraction principle is one of the most outstanding results in this theory. Since its appearance, several generalizations of this result have appeared in the literature. In 1976, Jungck [1] generalized this principle by considering two commutating mappings and proved a common fixed point theorem for these mappings. Afterwards, the commutative property of the mappings assumed by Jungck has been relaxed by introducing "weak" alternative notions as weak commutativity, (non)compatibility, R-weak commutativity, and weak compatibility, among others, which allowed extending several well-known common fixed point theorems for Lipschitz type of mapping pairs.

Here, we are going to establish existence and uniqueness results of common fixed point for a pair of contractive type of mappings whose contractive parameters are nonconstants and its contractive inequality is controlled by a positive function satisfying a stability condition at 0 (see (11)). As a particular case, our results are valid if we control the mentioned inequality by using the well-known altering distance functions [2]. To attain our goals, we will assume that the mappings under consideration are weakly compatible, which is a minimal noncommuting notion for contractive type of mapping pairs. Also, alternatively, we are going to assume that the pair of mappings satisfy some strong conditions like E. A. property and $C L R_{T}$ property.

In order to establish our results the following notions will be needed. A pair of self-mappings $(S, T)$ on a metric space $(M, d)$ is said to be compatible [3] if and only if $\lim _{n \rightarrow \infty} d\left(T S x_{n}, S T x_{n}\right)=0$, whenever $\left(x_{n}\right)_{n} \subset M$ is such that

$$
\lim _{n \rightarrow \infty} S x_{n}=\lim _{n \rightarrow \infty} T x_{n}=t
$$

for some $t \in M$. A pair of mappings $(S, T)$ will be said to be noncompatible if there exits at least one sequence $\left(x_{n}\right)_{n} \subset M$ such that $\lim _{n \rightarrow \infty} S x_{n}=\lim _{n \rightarrow \infty} T x_{n}=t$ for some $t \in M$, but $\lim _{n \rightarrow \infty} d\left(S T x_{n}, T S x_{n}\right)$ is either nonzero or nonexistent. A pair of self-mappings $(S, T)$ is said to satisfy the property (E.A.) [4] if there exists a sequence $\left(x_{n}\right)_{n} \subset M$ such that

$$
\lim _{n \rightarrow \infty} S x_{n}=\lim _{n \rightarrow \infty} T x_{n}=t
$$

for some $t \in M$.

A pair of self-mappings $(S, T)$ is said to satisfy the common limit in the range of $T$ property (in short $C L R_{T}$ ) [5] if there exists a sequence $\left(x_{n}\right)_{n} \subset M$ such that

$$
\lim _{n \rightarrow \infty} S x_{n}=\lim _{n \rightarrow \infty} T x_{n}=T t,
$$

for some $t \in M$. It may be observed that the $C L R_{T}$ property avoids the requirement of the condition of closeness of the ranges of the involved mappings. 
If $S$ is a self-map of a metric space $(M, d)$ then the set $O(x, S)=\left\{S^{n} x: n=0,1, \ldots\right\}$ is called the orbit of $S$ at $x$ and $S$ is called orbitally continuous if $z=\lim _{i} S^{m_{i}} x$ implies $S z=\lim _{i} S S^{m_{i}} x[6]$.

If $S$ and $T$ are self-maps of a metric space $(M, d)$ and if $\left\{x_{n}\right\}$ is a sequence in $(M, d)$ such that $S x_{n}=T x_{n+1}, n=$ $0,1,2, \ldots$, then the set $O\left(x_{0}, S, T\right)=\left\{S x_{n}, n=0,1,2, \ldots\right\}$ is called the $(S, T)$ orbit at $x_{0}$ and $S$ and $T$ are called orbitally continuous if $\lim _{n \rightarrow \infty} S x_{n}=\lim _{n \rightarrow \infty} T x_{n+1}=z$ implies $\lim _{n \rightarrow \infty} S T x_{n+1}=S z$ and $\lim _{n \rightarrow \infty} T S x_{n}=T z$.

A point $x \in M$ is called a coincidence point (CP) of $S$ and $T$ if $S x=T x$. The set of coincidence points of $S$ and $T$ will be denoted by $C(S, T)$. If $x \in C(S, T)$, then $w=S x=T x$ is called a point of coincidence (POC) of $S$ and $T$.

A pair of mappings $(S, T)$ is said to be nontrivially weakly compatible [7], whenever $x \in C(S, T) \neq \emptyset$ implies $S T x=T S x$. Finally, a pair of mappings $(S, T)$ is said to be occasionally weakly compatible (OWC) [8] if there exists some $x \in C(S, T)$ such that $S T x=T S x$.

Remark 1. We would like to show that weak compatibility is a necessary, hence minimal, condition for the existence of common fixed points of contractive type mapping pairs. Suppose $S$ and $T$ are a contractive type pair of self-mappings of a metric space $(M, d)$ having a common fixed point, say $z$; then $z=S z=T z$ and $S T z=T S z=S z=T z=z$. If possible, suppose that $S$ and $T$ are not weakly compatible. Then there exists a point $w$ in $M$ such that $S w=T w$ while $S T w \neq T S w$; we thus have $S w=T w$ and $S z=T z$ with $S w \neq S z$. This is not possible in view of contractive conditions. For example, if $S$ and $T$ satisfy the contractive condition

$$
d(S x, S y)<\max \{d(T x, T y), d(S x, T x), d(S y, T y)\},
$$

then we get

$$
\begin{aligned}
d(S w, S z) & <\max \{d(T w, T z), d(S w, T w), d(S z, T z)\} \\
& =d(S w, S z),
\end{aligned}
$$

a contradiction.

This shows that weak compatibility is a necessary, hence minimal, condition for the existence of common fixed points of contractive type mapping pairs.

The following result due to Babu and Sailaja in [9] will be useful in the sequel.

Lemma 2. Let $(M, d)$ be a metric space. Let $\left(x_{n}\right)$ be a sequence in $M$ such that

$$
\lim _{n \rightarrow \infty} d\left(x_{n}, x_{n+1}\right)=0 .
$$

If $\left(x_{n}\right)$ is not a Cauchy sequence in $M$, then there exist an $\varepsilon_{0}>0$ and sequences of integers positive $(m(k))$ and $(n(k))$ with

$$
m(k)>n(k)>k
$$

such that

$$
d\left(x_{m(k)}, x_{n(k)}\right) \geq \varepsilon_{0}, \quad d\left(x_{m(k)-1}, x_{n(k)}\right)<\varepsilon_{0}
$$

and
(i) $\lim _{k \rightarrow \infty} d\left(x_{m(k)-1}, x_{n(k)+1}\right)=\varepsilon_{0}$,
(ii) $\lim _{k \rightarrow \infty} d\left(x_{m(k)}, x_{n(k)}\right)=\varepsilon_{0}$,
(iii) $\lim _{k \rightarrow \infty} d\left(x_{m(k)-1}, x_{n(k)}\right)=\varepsilon_{0}$.

\section{The Class of Pairs of Mappings with Nonconstant Contractive Parameters}

In order to introduce the class of mappings which will be the focus of study of this paper, as in [10], we are going to use the functions $\alpha, \beta, \gamma: \mathbb{R}_{+} \rightarrow[0,1)$ which satisfy that $\alpha(t)+\beta(t)+$ $\gamma(t)<1$, for all $t \in \mathbb{R}_{+}$, and

$$
\begin{gathered}
\limsup _{s \rightarrow 0^{+}} \gamma(s)<1, \\
\limsup _{s \rightarrow t^{+}} \frac{\alpha(s)+\beta(s)}{1-\gamma(s)}<1, \quad \forall t>0 .
\end{gathered}
$$

Now, we introduce the following class of pair of contraction type of mappings.

Definition 3. Let $(M, d)$ be a metric space and let $S, T$ : $M \rightarrow M$ be mappings. The pair $(S, T)$ is called a $\psi-(\alpha, \beta, \gamma)-$ contraction pair if for all $x, y \in M$

$$
\begin{aligned}
\psi(d(S x, S y)) \leq & \alpha(d(T x, T y)) \psi(d(T x, T y)) \\
& +\beta(d(T x, T y)) \psi(d(S x, T x)) \\
& +\gamma(d(T x, T y)) \psi(d(S y, T y)),
\end{aligned}
$$

where $\alpha, \beta, \gamma: \mathbb{R}_{+} \rightarrow[0,1)$ are functions satisfying the conditions (9) and $\psi: \mathbb{R}_{+} \rightarrow \mathbb{R}_{+}:=[0,+\infty)$ is a continuous function satisfying that

$$
\psi\left(t_{n}\right) \rightarrow 0 \text { implies that } t_{n} \rightarrow 0 .
$$

Proposition 4. Let $S$ and $T$ be two self-maps on a metric space $(M, d)$. Let one assume that the pair $(S, T)$ is a $\psi-(\alpha, \beta, \gamma)$ contraction pair. If $S$ and $T$ have a POC in $M$ then it is unique.

Proof. Let $z \in M$ be a POC of the pair $(S, T)$. Then there exits $x \in M$ such that $S x=T x=z$. Suppose that, for some $v \in M$, $S v=T v=w$ with $z \neq w$. Then, by (10) we have

$$
\begin{aligned}
\psi(d(z, w))=\psi(d(S x, S v)) \leq & \alpha(d(T x, T v)) \psi(d(T x, T v)) \\
& +\beta(d(T x, T v)) \psi(d(S x, T x)) \\
& +\gamma(d(T x, T v)) \\
& \times \psi(d(S v, T v)) .
\end{aligned}
$$

It follows that

$$
\begin{aligned}
\psi(d(z, w)) \leq & \alpha(d(z, w)) \psi(d(z, w)) \\
& +\beta(d(z, w)) \psi(d(z, z)) \\
& +\gamma(d(z, w)) \psi(w, w) .
\end{aligned}
$$


Thus, we get

$$
\psi(d(z, w)) \leq \alpha(d(z, w)) \psi(d(z, w))<\psi(d(z, w))
$$

which is a contradiction. Therefore, $z=w$.

Proposition 5. Let $(M, d)$ be a metric space and let $S, T$ : $M \rightarrow M$ be mappings with $S(M) \subset T(M)$. If the pair $(S, T)$ is a $\psi$ - $(\alpha, \beta, \gamma)$-contraction pair, then for any $x_{0} \in M$, the sequence $\left(y_{n}\right)$ defined by

$$
y_{n}=S x_{n}=T x_{n+1}, \quad n=0,1, \ldots
$$

satisfies

(1) $\lim _{n \rightarrow \infty} d\left(y_{n}, y_{n+1}\right)=0$;

(2) $\left(y_{n}\right) \subset M$ is a Cauchy sequence in $M$.

Proof. To prove (1), let $x_{0} \in M$ be an arbitrary point. Since $S(M) \subset T(M)$, then there exists $x_{1} \in M$ such that $S x_{0}=T x_{1}$. By continuing this process inductively we obtain a sequence $\left(x_{n}\right)$ in $M$ such that

$$
y_{n}=S x_{n}=T x_{n+1} .
$$

Now, we have

$$
\begin{aligned}
& \psi\left(d\left(T x_{n+1}, T x_{n+2}\right)\right)=\psi\left(d\left(S x_{n}, S x_{n+1}\right)\right) \\
& \leq \alpha\left(d\left(T x_{n}, T x_{n+1}\right)\right) \psi\left(d\left(T x_{n}, T x_{n+1}\right)\right) \\
&+\beta\left(d\left(T x_{n}, T x_{n+1}\right)\right) \psi\left(d\left(S x_{n}, T x_{n}\right)\right) \\
&+\gamma\left(d\left(T x_{n}, T x_{n+1}\right)\right) \psi\left(d\left(S x_{n+1}, T x_{n+1}\right)\right) .
\end{aligned}
$$

It follows that

$$
\begin{aligned}
& \psi\left(d\left(T x_{n+1}, T x_{n+2}\right)\right) \\
& \leq \alpha\left(d\left(T x_{n}, T x_{n+1}\right)\right) \psi\left(d\left(T x_{n}, T x_{n+1}\right)\right) \\
&+\beta\left(d\left(T x_{n}, T x_{n+1}\right)\right) \psi\left(d\left(T x_{n+1}, T x_{n}\right)\right) \\
&+\gamma\left(d\left(T x_{n}, T x_{n+1}\right)\right) \psi\left(d\left(T x_{n+2}, T x_{n+1}\right)\right) .
\end{aligned}
$$

Therefore, we obtain

$$
\begin{aligned}
& \psi\left(d\left(T x_{n+1}, T x_{n+2}\right)\right) \\
& \leq \frac{\alpha\left(d\left(T x_{n}, T x_{n+1}\right)\right)+\beta\left(d\left(T x_{n}, T x_{n+1}\right)\right)}{1-\gamma\left(d\left(T x_{n}, T x_{n+1}\right)\right)} \\
& \times \psi\left(d\left(T x_{n}, T x_{n+1}\right)\right),
\end{aligned}
$$

from which, together with (9), we conclude that

$$
\psi\left(d\left(T x_{n+1}, T x_{n+2}\right)\right)<\psi\left(d\left(T x_{n}, T x_{n+1}\right)\right) .
$$

Thus, $\left(\psi\left(d\left(T x_{n}, T x_{n+1}\right)\right)\right)_{n}$ is a nondecreasing sequence, bounded below by zero, and so converges to $a \geq 0$. Now, if $a>0$ then by taking lim sup from both sides of the above inequality we have a contradiction. Thus, $a=0$. Now, from condition (11) we conclude that $d\left(T x_{n}, T x_{n+1}\right) \rightarrow 0$.
To prove (2), we are going to suppose that $\left(y_{n}\right) \subset T(M)$ is not a Cauchy sequence. Then, from Lemma 2 there exist $\varepsilon>0$ and sequences $(m(k))$ and $(n(k))$ with $m(k) \geq n(k)>k$ such that

$$
\begin{gathered}
\lim _{k \rightarrow \infty} d\left(T x_{m(k)}, T x_{n(k)}\right)=\varepsilon, \\
\lim _{k \rightarrow \infty} d\left(T x_{m(k)-1}, T x_{n(k)-1}\right)=\varepsilon .
\end{gathered}
$$

In this way we have

$$
\begin{aligned}
0<\psi(\varepsilon)=\limsup _{k \rightarrow \infty} \psi\left(d\left(T x_{m(k)}, T x_{n(k)}\right)\right) \\
=\limsup _{k \rightarrow \infty} \psi\left(d\left(S x_{m(k)-1}, S x_{n(k)-1}\right)\right) \\
\leq \limsup _{k \rightarrow \infty} \alpha\left(d\left(T x_{m(k)-1}, T x_{n(k)-1}\right)\right) \\
\quad \times \psi\left(d\left(T x_{m(k)-1}, T x_{n(k)-1}\right)\right) \\
\quad+\limsup _{k \rightarrow \infty} \beta\left(d\left(T x_{m(k)-1}, T x_{n(k)-1}\right)\right) \\
\quad \times \psi\left(d\left(S x_{m(k)-1}, T x_{m(k)-1}\right)\right) \\
\quad+\limsup _{k \rightarrow \infty} \gamma\left(d\left(T x_{m(k)-1}, T x_{n(k)-1}\right)\right) \\
\quad \times \psi\left(d\left(S x_{n(k)-1}, T x_{n(k)-1}\right)\right) \\
\leq \limsup _{s \rightarrow \varepsilon}(s) \limsup _{k \rightarrow \infty}\left(d\left(T x_{m(k)-1}, T x_{n(k)-1}\right)\right) \\
\quad+\limsup _{s \rightarrow \varepsilon} \beta(s) \limsup _{k \rightarrow \infty} \psi\left(d\left(T x_{m(k)}, T x_{m(k)-1}\right)\right) \\
+\limsup _{s \rightarrow \varepsilon} \gamma(s) \limsup _{k \rightarrow \infty} \psi\left(d\left(T x_{n(k)}, T x_{n(k)-1}\right)\right) \\
<\psi(\varepsilon),
\end{aligned}
$$

which is a contradiction; hence, $\left(T x_{n}\right)$ is a Cauchy sequence in $M$.

\section{On the Existence and Uniqueness of Common Fixed Points}

In this section we prove our main results concerning the existence and uniqueness of common fixed points for a $\psi-(\alpha, \beta, \gamma)$-contraction pair of mappings without continuity requirement.

Theorem 6. Let $S$ and $T$ be self-maps on a complete metric space $(M, d)$ such that

(i) $S(M) \subset T(M)$;

(ii) the pair $(S, T)$ is a $\psi$ - $(\alpha, \beta, \gamma)$-contraction pair. 
Then,

(1) the pair $(S, T)$ has a unique POC;

(2) if $S$ and $T$ are orbitally continuous and if the pair $(S, T)$ is compatible, then $S$ and $T$ have a unique common fixed point.

Proof. Let $y_{n}=S x_{n}=T x_{n+1}, n=0,1, \ldots$, be the Cauchy sequence defined in Proposition 5 which, as was proved, satisfies that $\left(y_{n}\right)=\left(T x_{n+1}\right) \subset T(M)$. Since $(M, d)$ is complete, there exists a point $z$ in $(M, d)$ such that

$$
\lim _{n \rightarrow \infty} y_{n}=\lim _{n \rightarrow \infty} S x_{n}=\lim _{n \rightarrow \infty} T x_{n+1}=z .
$$

Compatibility and orbital continuity of $S$ and $T$ imply $S z=T z$; hence $z$ is a POC of $S$ and T. From Proposition 4 we conclude that $z$ is the unique POC.

On the other hand, since the pair $(S, T)$ is compatible and compatible mappings commute at their coincidence point, $S S z=S T z=T S z=T T z$. Using (ii), we get

$$
\begin{aligned}
\psi(d(S z, S S z)) \leq & \alpha(d(T z, T S z)) \psi(d(T z, T S z)) \\
& +\beta(d(T z, T S z)) \psi(d(S z, T z)) \\
& +\gamma(d(T z, T S z)) \psi(d(S S z, T S z)) \\
= & \alpha(d(T z, T S z)) \psi(d(S z, S S z)) ;
\end{aligned}
$$

that is, $S z=S S z$. Hence, $S z=S S z=T S z$, and $S z$ is a common fixed point of the pair $(S, T)$. The uniqueness of the common fixed point follows easily.

Theorem 7. Let $S$ and $T$ be self-mappings on a metric space $(M, d)$ such that

(i) $S(M) \subset T(M)$;

(ii) $T(M) \subset M$ is a complete subspace of $M$;

(iii) the pair $(S, T)$ is a $\psi$ - $(\alpha, \beta, \gamma)$-contraction pair.

Then,

(1) the pair $(S, T)$ has a unique POC;

(2) if the pair $(S, T)$ is nontrivially weakly compatible, then $S$ and $T$ have a unique common fixed point.

Proof. Let $y_{n}=S x_{n}=T x_{n+1}, n=0,1, \ldots$, be the Cauchy sequence defined in Proposition 5 which, as was proved, satisfies that $\left(y_{n}\right)=\left(T x_{n+1}\right) \subset T(M)$. Since $T(M) \subset M$ is a complete subspace of $M$, then there exists $z \in T(M)$ such that

$$
\lim _{n \rightarrow \infty} y_{n}=\lim _{n \rightarrow \infty} S x_{n}=\lim _{n \rightarrow \infty} T x_{n+1}=z
$$

and thus we can find $u \in M$ such that $T u=z$. Now, we are going to assume that $S u \neq z$. Then,

$$
\begin{aligned}
\psi\left(d\left(S x_{n+1}, S u\right)\right) \leq & \alpha\left(d\left(T x_{n+1}, T u\right)\right) \psi\left(d\left(T x_{n+1}, T u\right)\right) \\
& +\beta\left(d\left(T x_{n+1}, T u\right)\right) \psi\left(d\left(S x_{n+1}, T x_{n+1}\right)\right) \\
& +\gamma\left(d\left(T x_{n+1}, T u\right)\right) \psi(S u, T u) .
\end{aligned}
$$

Letting $n \rightarrow \infty$, we obtain

$$
\begin{aligned}
\psi(d(S u, z)) \leq & \limsup _{n \rightarrow \infty} \alpha\left(d\left(T x_{n+1}, T u\right)\right) \psi(d(z, T u)) \\
& +\limsup _{n \rightarrow \infty} \beta\left(d\left(T x_{n+1}, T u\right)\right) \psi(d(z, z)) \\
& +\limsup _{n \rightarrow \infty} \gamma\left(d\left(T x_{n+1}, T u\right)\right) \psi(d(S u, T u)) \\
< & \psi(d(S u, z))
\end{aligned}
$$

which is a contradiction; therefore $S u=z$; hence $z$ is a POC of $S$ and $T$. The theorem now follows easily using arguments similar to those which have been used in the corresponding part of Theorem 6 .

Theorem 7 is also true if we replace conditions (i) and (ii) (of Theorem 7) by a single condition. In the next theorem $\overline{S M}$ denotes the closure of the range of the mapping S.

Theorem 8. Let $S$ and $T$ be self-mappings on a metric space $(M, d)$ such that

(i) $\overline{S M} \subset T(M)$;

(ii) the pair $(S, T)$ is a $\psi$ - $(\alpha, \beta, \gamma)$-contraction pair.

Then,

(1) the pair $(S, T)$ has a unique POC;

(2) if the pair $(S, T)$ is nontrivially weakly compatible, then $S$ and $T$ have a unique common fixed point.

Remark 9. Notice that in Theorem 7 we cannot replace nontrivially weakly compatible mappings by OWC. In fact, under the $\psi$ - $(\alpha, \beta, \gamma)$-contraction pair of Theorem 6 , assumption of OWC and the existence of a unique common fixed point are equivalent conditions. To see this, first suppose that $A$ and $S$ satisfy the $\psi$ - $(\alpha, \beta, \gamma)$-contraction condition of Theorem 6 above. If $A$ and $S$ have a common fixed point, say $z$, then $=A z=S z, \sqrt{i} A S z=S A z=z$, and $A$ and $S$ are, therefore, OWC mappings. On the other hand, if $A$ and $S$ are OWC mappings such that $A x=S x$ and $A S x=S A x=A A x=S S x$ for some $x$ then, using $\psi$ - $(\alpha, \beta, \gamma)$-contraction condition, we get

$$
\begin{aligned}
\psi(d(S x, S S x)) \leq & \alpha(d(T x, T S x)) \psi(d(T x, T S x)) \\
& +\beta(d(T x, T S x)) \psi(d(S x, T x)) \\
& +\gamma(d(T x, T S x)) \psi(d(S S x, T S x)) \\
= & \alpha(d(T x, T S x)) \psi(d(S x, S S x))
\end{aligned}
$$

That is, $S x=S S x$. Since $\psi-(\alpha, \beta, \gamma)$-contraction condition excludes the existence of two coincidence points $x, y$ for $A$ and $S$, we get $S x=S S x(=A S x)$. This means that $S x=A x$ is a common fixed point of $A$ and $S$. Therefore, one should be really careful before using OWC under any contractive conditions (see, also, [11]). 
Theorem 10. Let $(M, d)$ be a metric space and $S, T: M \rightarrow$ $M$ nontrivially weakly compatible mappings satisfying the property (E.A.). Let one suppose that the pair $(S, T)$ is a $\psi-(\alpha, \beta, \gamma)$-contraction pair. If $T(M) \subset M$ is closed, then $S$ and $T$ have a unique common fixed point.

Proof. Since the pair $(S, T)$ satisfies the property (E.A.), then there exists a sequence $\left(x_{n}\right) \subset M$ such that

$$
\lim _{n \rightarrow \infty} S x_{n}=\lim _{n \rightarrow \infty} T x_{n}=z
$$

for some $z \in M$. Since $T(M)$ is closed, then $z \in T(M)$ and $z=T u$ for some $u \in M$. As in the proof of Theorem 7, we can prove that $z=T u=S u$ and that $z$ is the unique POC of $S$ and $T$. The rest of the proof of the theorem follows from Theorem 6 .

Remark 11. Since two noncompatible self-mappings on a metric space $(M, d)$ satisfy the property (E.A.), then the conclusion of Theorem 10 remains valid if we consider $S$ and $T$ noncompatible self-mappings.

In the next theorem we drop closeness of the range of mapping and replace property (E.A.) with $C L R_{T}$ property.

Theorem 12. Let $(M, d)$ be a metric space and $S, T: M \rightarrow M$ satisfying the $C L R_{T}$ property. Let us suppose that the pair $(S, T)$ is a $\psi-(\alpha, \beta, \gamma)$-contraction pair. If the pair $(S, T)$ is nontrivially weakly compatible, then $S$ and $T$ have a unique common fixed point.

Proof. Since the pair $(S, T)$ satisfies the $C L R_{T}$ property, then there exists a sequence $\left(x_{n}\right) \subset M$ such that

$$
\lim _{n \rightarrow \infty} S x_{n}=\lim _{n \rightarrow \infty} T x_{n}=T z
$$

for some $z \in M$.

The rest of the proof of the theorem follows easily.

\section{Controlling by Altering Distance Functions}

In 1976, Delbosco [12] initiated the study of fixed point for contractive conditions using altering distance functions; however, his study was limited to some power functions only. Subsequently, his result was extended by Skof [13] and Khan et. al [2] in 1977 and 1984, respectively. Since then, it has been used to solve several problems in the metric fixed point theory (see, e.g., [14-21]).

Definition 13. A function $\psi: \mathbb{R}_{+} \rightarrow \mathbb{R}_{+}$is called an altering distance function if the following properties are satisfied:

$\left(\Psi_{1}\right) \psi(t)=0$ if and only if $t=0$;

$\left(\Psi_{2}\right) \psi$ is monotonically nondecreasing;

$\left(\Psi_{3}\right) \psi$ is continuous.

By $\Psi$ we are going to denote the set of all the altering distance functions.

Since every nondecreasing map $\psi$ satisfies (11) (but the converse is not true), then all the previous results are valid, in particular, if we replace functions satisfying the condition (11) for altering distance functions.

On the other hand, in 2002, Branciari [22] extended the Banach-Caccioppoli theorem by using some Lebesgue integrable functions. Since then, several well-known fixed point criteria for contractive type of mappings have been generalized in this way. See, for example, $[5,10,16,17,23-$ 27] and a lot of references therein. In 2009, Jachymski [28] showed that most contractive conditions of integral type given by many authors are mere consequences of classical known ones (see [28] and references therein).

By $\Phi$ is denoted the set of all mappings $\phi: \mathbb{R}_{+} \rightarrow \mathbb{R}_{+}$ satisfying the following conditions:

$\left(\Phi_{1}\right) \phi$ is a Lebesgue integrable mapping which is summable on each compact subset of $\mathbb{R}_{+}$;

$\left(\Phi_{2}\right) \phi$ is nonnegative;

$\left(\Phi_{3}\right)$ for each $\varepsilon>0, \int_{0}^{\varepsilon} \phi(t) d t>0$.

A relation between these two classes of functions $\Psi$ and $\Phi$ is given in the following result $([29,30])$.

Lemma 14. For each $\varphi \in \Phi$, the function $\psi_{0}: \mathbb{R}_{+} \rightarrow \mathbb{R}_{+}$ defined by $\psi_{0}(s)=\int_{0}^{s} \varphi(t) d t, s \in \mathbb{R}_{+}$is such that $\psi_{0} \in \Psi$.

In this way, additionally to the class of $\psi$ - $(\alpha, \beta, \gamma)$ contraction pairs, we can consider a type of pair of mappings satisfying the following inequality contraction of integral type:

$$
\begin{aligned}
\int_{0}^{\psi(d(S x, S y))} \varphi(t) d t \\
\leq \alpha(d(T x, T y)) \int_{0}^{\psi(d(T x, T y))} \varphi(t) d t+\beta(d(T x, T y)) \\
\quad \times \int_{0}^{d(S x, T x)} \varphi(t) d t+\gamma(d(T x, T y)) \\
\quad \times \int_{0}^{\psi(d(S y, T y))} \varphi(t) d t
\end{aligned}
$$

for all $x, y \in M$, where $\psi \in \Psi, \varphi \in \Phi$, and $\alpha, \beta, \gamma$ are functions satisfying (9). Since this class can be rewritten as

$$
\begin{aligned}
\psi_{0}(\psi(d(S x, S y))) \leq & \alpha(d(T x, T y)) \psi_{0}(\psi(d(T x, T y))) \\
& +\beta(d(T x, T y)) \psi_{0}(\psi(d(S x, T x))) \\
& +\gamma(d(T x, T y)) \psi_{0}(\psi(d(S y, T y)))
\end{aligned}
$$

for all $x, y \in M$, where $\psi_{0} \in \Psi$ is the function defined in Lemma 14, then we have that all the conclusions given for $\psi-(\alpha, \beta, \gamma)$-contraction pairs are automatically valid for pair of mappings satisfying the inequality contraction (31). 


\section{Conclusions and Examples}

Notice that due to the minor restrictions on the functions involved in the definition of the class of $\psi-(\alpha, \beta, \gamma)$ contraction pairs and the minimal commutative requirements of the mappings, our results extend several common fixed point theorems for classes of well-known contractive type of mappings, including various classes of contractive mappings with inequalities controlled by altering distance functions as well as contractive mappings of the integral type. Even more, the mappings $T$ and $S$ considered here are not necessarily continuous, so in this way our results are more general compared with other results in this line of research.

Next, we are going to show some examples in support of our results.

Example 15. Let $M=[0,1]$ be equipped with the Euclidean metric. We consider the following mappings: $S, T: M \rightarrow M$ defined by $S x=x / 16$ and $T x=x / 2$ for all $x \in M$. Let $\alpha, \beta, \gamma$ : $\mathbb{R}_{+} \rightarrow[0,1)$ defined by $\alpha(t)=\beta(t)=1 / 4$ and $\gamma(t)=1 / 8$ for all $x \in \mathbb{R}_{+}$and $\psi: \mathbb{R}_{+} \rightarrow \mathbb{R}_{+}$given by the formula $\psi(t)=t^{2}$, $t \in \mathbb{R}_{+}$.

Notice that $\psi \in \Psi$ and the functions $\alpha, \beta, \gamma$ satisfy the conditions (9); also note that $S(M) \subset T(M)$ and $T(M)$ is a complete subspace of $M$. Moreover, it is not difficult to show that the pair $(S, T)$ is a $\psi-(\alpha, \beta, \gamma)$-contraction pair. Besides, $C(S, T)=\{0\}$ and $S T 0=T S 0=0$, which mean that $(S, T)$ is nontrivially weakly compatible. Then, Theorem 7 guarantees that $w=0$ is the unique common fixed point of $S$ and $T$.

Example 16. As in the example before, $M=[0,1]$ with the usual metric. We define the self-maps $S, T$ on $M$ by

$$
S x= \begin{cases}0, & \text { if } 0 \leq x \leq \frac{1}{2} \\ \frac{1}{16}, & \text { if } \frac{1}{2}<x \leq 1\end{cases}
$$

and $T x=x / 2$ for all $x \in M$. Let $\alpha, \beta, \gamma: \mathbb{R}_{+} \rightarrow[0,1)$ defined as follows:

$$
\alpha(t)=\frac{1}{8}, \quad \beta(t)=\gamma(t)=\frac{1}{4}, \quad \forall t \in \mathbb{R}_{+} .
$$

Let $\psi: \mathbb{R}_{+} \rightarrow \mathbb{R}_{+}$defined by $\psi(t)=t^{2}, t \in \mathbb{R}_{+}$. Then, the pair $(S, T)$ is a $\psi-(\alpha, \beta, \gamma)$-contraction pair satisfying the hypotheses of Theorem 6; thus $w=0$ is the unique POC and moreover the unique common fixed point of $S$ and $T$.

Example 17. Let $M=[1 / 2,1]$ with the usual metric on $\mathbb{R}$. In this case we consider the mappings $S, T: M \rightarrow M$ defined by

$$
\begin{gathered}
S x= \begin{cases}\frac{1}{2}, & \text { if } \frac{1}{2} \leq x<\frac{2}{3} \\
1-\frac{1}{2} x, & \text { if } \frac{2}{3} \leq x \leq 1,\end{cases} \\
T x= \begin{cases}1, & \text { if } \frac{1}{2} \leq x<\frac{2}{3} \\
x, & \text { if } \frac{2}{3} \leq x \leq 1 .\end{cases}
\end{gathered}
$$

Let $\psi: \mathbb{R}_{+} \rightarrow \mathbb{R}_{+}$given by the formula $\psi(t)=t^{2}, t \in \mathbb{R}_{+}$. $\alpha, \beta, \gamma: \mathbb{R}_{+} \rightarrow[0,1)$ given by $\alpha(t)=\beta(t)=1 / 4, \gamma(t)=1 / 8$, $t \in \mathbb{R}_{+}$. Notice that $C(S, T)=\{2 / 3\}$ and $S T(2 / 3)=T S(2 / 3)$. Moreover, $w=2 / 3$ is the unique POC of $S$ and $T$; thus the pair $(S, T)$ is nontrivially weakly compatible. On the other hand, by considering the sequence $x_{n}=2 / 3+1 / n, n \geq 4$ in $M$, it is clear that the pair $(S, T)$ satisfies the property (E.A.). Finally, it is easy to show that in fact the mappings $S, T, \psi, \alpha, \beta$ and $\gamma$ satisfy all the hypotheses of Theorem 10, so $w=2 / 3$ is the unique common fixed point of $S$ and $T$.

\section{Conflict of Interests}

The authors declare that there is no conflict of interests regarding the publication of this paper.

\section{Acknowledgments}

The authors are thankful to the referees for the very constructive comments and suggestions that led to an improvement of the paper. E. M. Rojas is sponsored by Pontificia Universidad Javeriana under Grant no. 000000000005781.

\section{References}

[1] G. Jungck, "Commuting mappings and fixed points," The American Mathematical Monthly, vol. 83, no. 4, pp. 261-263, 1976.

[2] M. S. Khan, M. Swaleh, and S. Sessa, "Fixed point theorems by altering distances between the points," Bulletin of the Australian Mathematical Society, vol. 30, no. 1, pp. 1-9, 1984.

[3] G. Jungck, "Compatible mappings and common fixed points," International Journal of Mathematics and Mathematical Sciences, vol. 9, no. 4, pp. 771-779, 1986.

[4] M. Aamri and D. El Moutawakil, "Some new common fixed point theorems under strict contractive conditions," Journal of Mathematical Analysis and Applications, vol. 270, no. 1, pp. 181188, 2002.

[5] W. Sintunavarat and P. Kumam, "Gregus type fixed points for a tangential multi-valued mappings satisfying contractive conditions of integral type," Journal of Inequalities and Applications, vol. 2011, article 3, 2011.

[6] L. B. Ćirić, "On some maps with a nonunique fixed point," Publications de l'Institut Mathématique, vol. 17(31), pp. 52-58, 1974.

[7] G. Jungck and B. E. Rhoades, "Fixed point theorems for occasionally weakly compatible mappings," Fixed Point Theory, vol. 7, no. 2, pp. 287-296, 2006.

[8] M. A. Al-Thagafi and N. Shahzad, "Generalized I-nonexpansive selfmaps and invariant approximations," Acta Mathematica Sinica, vol. 24, no. 5, pp. 867-876, 2008.

[9] G. V. R. Babu and P. D. Sailaja, "A fixed point theorem of generalized weakly contractive maps in orbitally complete metric spaces," Thai Journal of Mathematics, vol. 9, no. 1, pp. 110, 2011.

[10] Z. Liu, X. Li, S. Minkan, and S. Y. Cho, "Fixed point theorems for mappings satisfying contractive conditions of integral type and applications," Fixed Point Theory and Applications, vol. 2011, article 64, 2011. 
[11] R. P. Pant and R. K. Bisht, "Occasionally weakly compatible mappings and fixed points," Bulletin of the Belgian Mathematical Society. Simon Stevin, vol. 19, no. 4, pp. 655-661, 2012.

[12] D. Delbosco, "Un'estensione di un teorema sul punto fisso di S. Reich," Rendiconti del Seminario Matematico Università e Politecnico di Torino, vol. 35, pp. 233-238, 1976-1977.

[13] F. Skof, "Teoremi di punto fisso per applicazioni negli spazi metrici," Atti della Accademia delle Scienze di Torino. Classe di Scienze Fisiche, Matematiche e Naturali, vol. 111, no. 3-4, pp. 323329, 1977.

[14] P. N. Dutta and B. S. Choudhury, "A generalisation of contraction principle in metric spaces," Fixed Point Theory and Applications, vol. 2008, Article ID 406368, 8 pages, 2008.

[15] P. N. Dutta, B. S. Choudhury, and K. Das, "Some fixed point results in Menger spaces using a control function," Surveys in Mathematics and Its Applications, vol. 4, pp. 41-52, 2009.

[16] J. R. Morales and E. M. Rojas, "Some generalizations of Jungck's fixed point theorem," International Journal of Mathematics and Mathematical Sciences, vol. 2012, Article ID 213876, 19 pages, 2012.

[17] J. R. Morales and E. Rojas, "Some fixed point theorems by altering distance functions," Palestine Journal of Mathematics, vol. 1, no. 2, pp. 110-116, 2012.

[18] S. V. R. Naidu, "Some fixed point theorems in metric spaces by altering distances," Czechoslovak Mathematical Journal, vol. 53, no. 1, pp. 205-212, 2003.

[19] H. K. Nashine, B. Samet, and J. K. Kim, "Fixed point results for contractions involving generalized altering distances in ordered metric spaces," Fixed Point Theory and Applications, vol. 2011, article 5, 2011.

[20] K. P. R. Sastry and G. V. R. Babu, "Some fixed point theorems by altering distances between the points," Indian Journal of Pure and Applied Mathematics, vol. 30, no. 6, pp. 641-647, 1999.

[21] J. Jachymski, "Equivalent conditions for generalized contractions on (ordered) metric spaces," Nonlinear Analysis: Theory, Methods \& Applications, vol. 74, no. 3, pp. 768-774, 2011.

[22] A. Branciari, "A fixed point theorem for mappings satisfying a general contractive condition of integral type," International Journal of Mathematics and Mathematical Sciences, vol. 29, no. 9, pp. 531-536, 2002.

[23] A. Aliouche, "A common fixed point theorem for weakly compatible mappings in symmetric spaces satisfying a contractive condition of integral type," Journal of Mathematical Analysis and Applications, vol. 322, no. 2, pp. 796-802, 2006.

[24] H. Aydi, "A common fixed point result by altering distances involving a contractive condition of integral type in partial metric spaces," Demonstratio Mathematica, vol. 46, no. 2, pp. 383-394, 2013.

[25] Y. J. Cho, B. E. Rhoades, R. Saadati, B. Samet, and W. Shatanawi, "Nonlinear coupled fixed point theorems in ordered generalized metric spaces with integral type," Fixed Point Theory and Applications, vol. 2012, article 8, 2012.

[26] B. E. Rhoades, "Two fixed-point theorems for mappings satisfying a general contractive condition of integral type," International Journal of Mathematics and Mathematical Sciences, vol. 2003, no. 63, pp. 4007-4013, 2003.

[27] B. Samet and C. Vetro, "An integral version of Ćirić's fixed point theorem," Mediterranean Journal of Mathematics, vol. 9, no. 1, pp. 225-238, 2012.

[28] J. Jachymski, "Remarks on contractive conditions of integral type," Nonlinear Analysis: Theory, Methods \& Applications, vol. 71, no. 3-4, pp. 1073-1081, 2009.
[29] M. Abbas and M. A. Khan, "Common fixed point theorem of two mappings satisfying a generalized weak contractive condition," International Journal of Mathematics and Mathematical Sciences, vol. 2009, Article ID 131068, 9 pages, 2009.

[30] V. Popa and M. Mocanu, "Altering distance and common fixed points under implicit relations," Hacettepe Journal of Mathematics and Statistics, vol. 38, no. 3, pp. 329-337, 2009. 


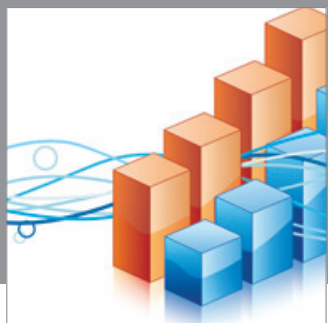

Advances in

Operations Research

mansans

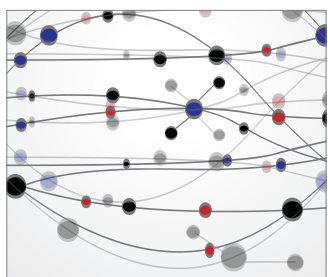

The Scientific World Journal
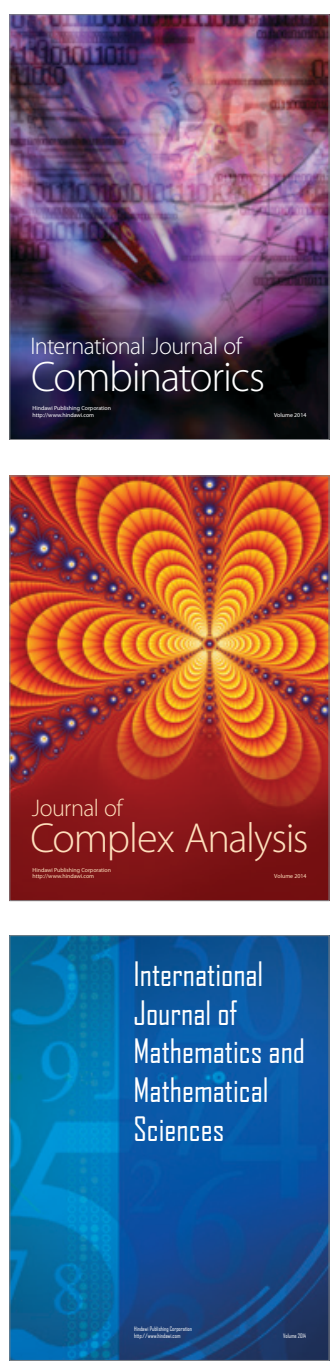
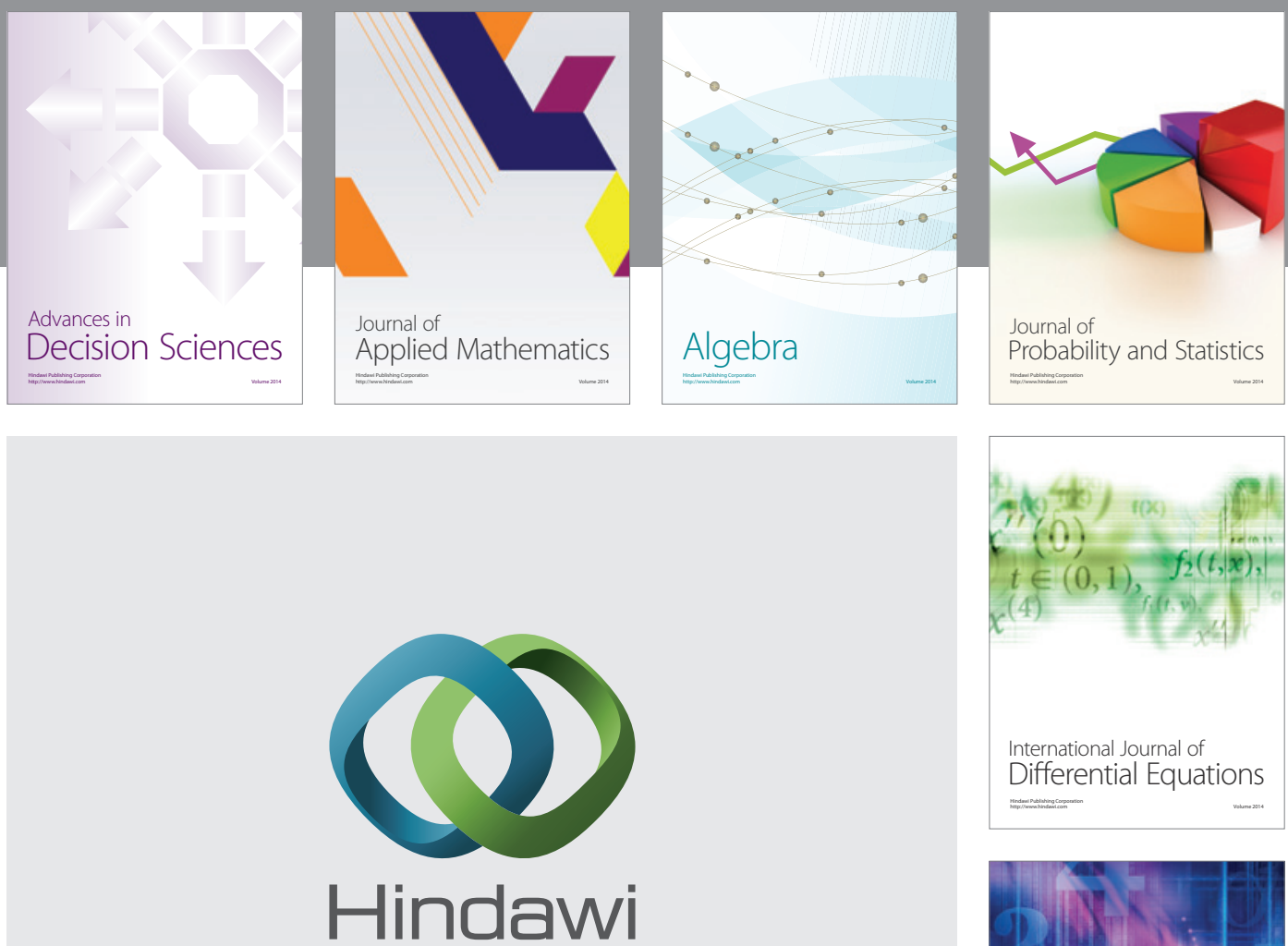

Submit your manuscripts at http://www.hindawi.com
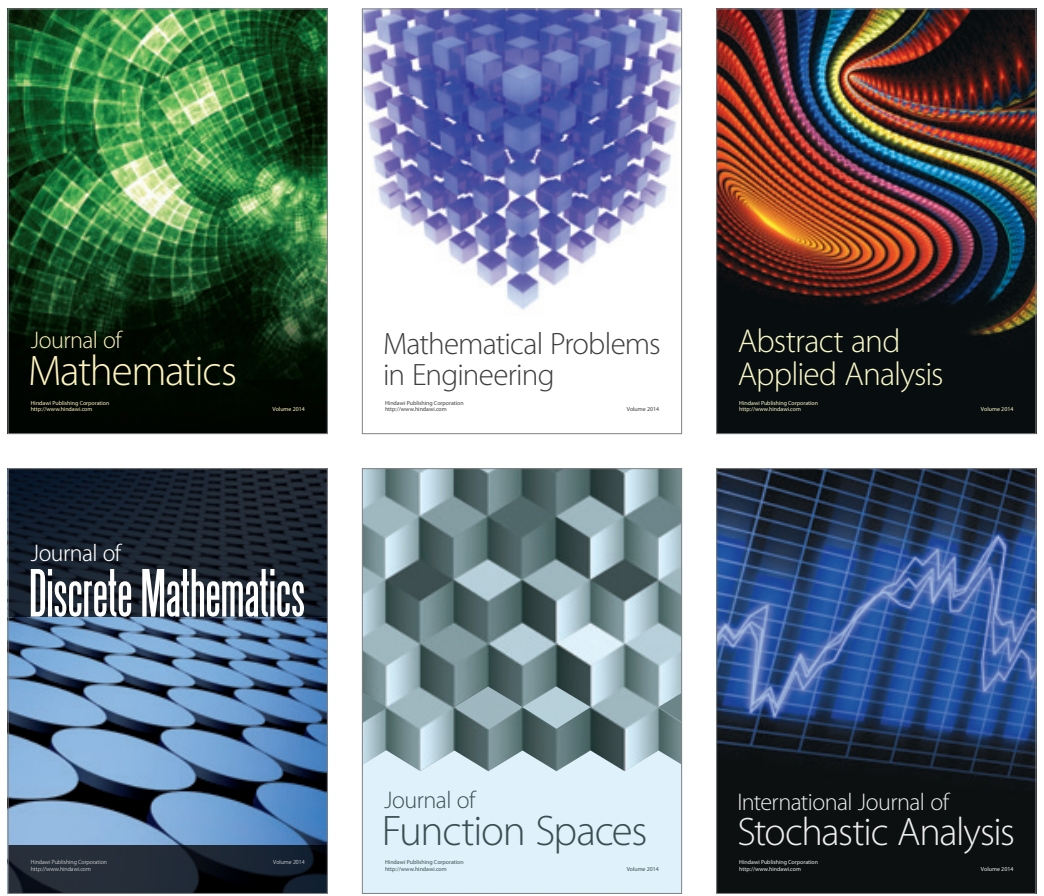

Journal of

Function Spaces

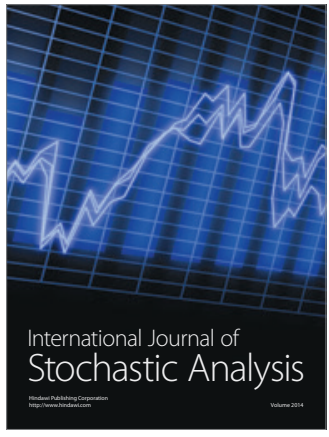

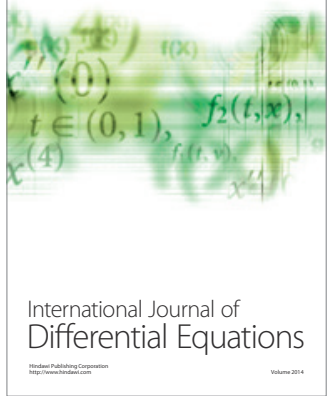
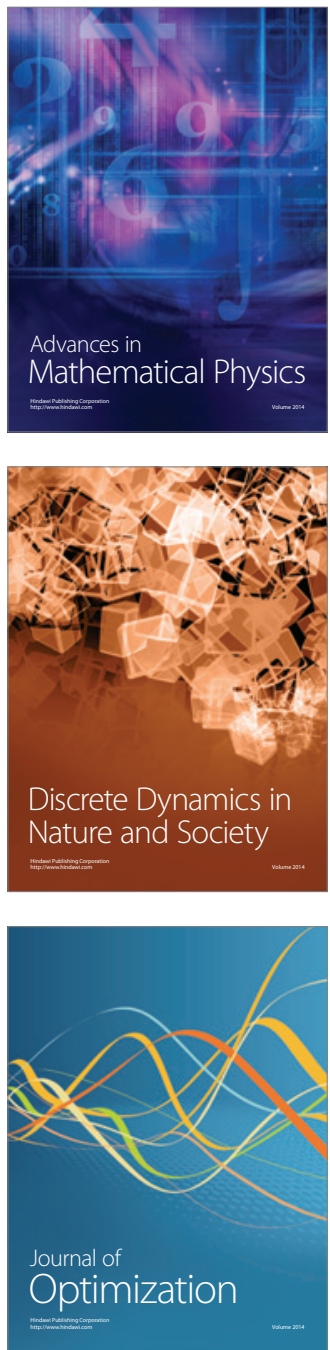